\title{
Low-dose CT in COVID-19 outbreak: radiation safety, image wisely, and image gently pledge
}

\author{
Salar Tofighi ${ }^{1} \cdot$ Saeideh Najafi ${ }^{1}$ (D) Sean K. Johnston ${ }^{1} \cdot$ Ali Gholamrezanezhad ${ }^{1}$
}

Received: 19 April 2020 / Accepted: 29 April 2020 / Published online: 10 May 2020

(C) American Society of Emergency Radiology 2020

By April 24, 2020, COVID-19, which refers to severe acute respiratory syndrome coronavirus 2 (SARS-CoV-2) infection, has been spread to almost all countries, contaminated more than 2,600,000 individuals, and led to more than 180,000 deaths worldwide [1]. COVID-19 is caused by a type of coronavirus which infects both animals and human and predominantly involves the respiratory system. The virus was initially introduced to humans from bats in Wuhan, China [2]. Despite the Chinese government initiating the most aggressive disease containment effort in history [3], the disease has rapidly grown to a pandemic. So far in the USA, more than 869,000 have been infected and almost 50,000 died [4] since the first case was recognized on January 20, 2020 [5].

COVID-19 when symptomatic presents with pneumonia or upper respiratory infection. Fever is observed in almost all (8398\%) patients and cough (mostly dry) is manifested in the majority of cases (46-82\%). About one-third of patients (31\%) may present with shortness of breath. Myalgia and fatigue may also present (11-44\%). Pharyngitis, productive cough, hemoptysis, headache, and GI symptoms are less common manifestations. Of infected individuals, $80 \%$ are asymptomatic or

Please note that due to the time sensitive nature of the work presented in this article, standard peer-review has been bypassed to ensure rapid publication. This contribution has been directly assessed by the Editor-inChief.

Saeideh Najafi

Saeideh.njf@gmail.com

Salar Tofighi

salar.tofighi@usc.edu

Sean K. Johnston

sean.johnston@med.usc.edu

Ali Gholamrezanezhad

ali.gholamrezanezhad@med.usc.edu

1 Keck School of Medicine, University of Southern California, 1500, San Pablo St., Room 2250, Los Angeles, CA 90033, USA only manifest mild symptoms. The average age of patients has been in their 50s with a slight male dominance [6-8]. The mean incubation period is about 5 days after exposure but can be as long as 19 days during which the infected individual is asymptomatic and suspected to be able to transmit disease [6]. Lymphopenia, elevated lactate dehydrogenase, and ferritin are the most common laboratory findings observed in COVID-19 [6].

Definitive diagnosis is made by the detection of viral RNA in specimens collected from patients' respiratory secretions using reverse transcriptase-polymerase chain reactions (RTPCR) that are performed using pre-prepared laboratory kits [9]. Since COVID-19 emerged very recently, the efficacy and accuracy of available kits have not been evaluated thoroughly and the sensitivity of currently produced kits has been reported to be as low as 59\% in some studies [9]. With the increasing emergent need, governments have encountered a shortage of kits and problems with their distribution, administration, and storage. For instance, the kits stored in the Atlanta CDC facility are reported to be contaminated with Coronavirus [10]. The diagnosis of the first case of COVID19 in Washington state was delayed due to issues with the results of RT-PCR kits distributed by the CDC. Meanwhile, the suspected patient and exposed healthcare providers were not isolated and freely moved about potential exposing many other individuals to the disease [11].

\section{CT in the diagnostic approach to COVID-19}

The rapid widespread expansion of COVID-19 cases has raised concerns about possible disease containment and has made control of further spread of the disease the priority in the management of COVID-19 by WHO and CDC to prevent a pandemic [9]. Bill Gates in his article published in NEJM compared COVID-19 pandemic with the 1918 influenza pandemic and indicated that COVID-19 may evoke a worse pandemic because it has a higher rate of contagiousness and kills 
both young and elderly [12]. Since there is currently no vaccine for COVID-19, successful disease containment is contingent upon rapid identification of infected individuals to isolate them and their close contacts $[9,13]$. The urgent need for an alternative diagnostic than RT-PCR kits has given rise to the possibility of utilization of chest CT findings as a diagnostic aid and a screening tool in suspected patients [13].

In a review of the worldwide approach to the management of COVID-19 outbreak, authors reported that many countries are currently using CT scan as a screening tool for suspected patients due to the lack of resources (under review). Recently, a large study from Wuhan has suggested application of CT scan as an alternative diagnostic method which as of today demonstrated higher sensitivity than existing RT-PCR kits [9] despite that it has not yet been approved by major radiology associations. CT scan is a widely available modality and can readily be performed. In this study, it was reported that $88 \%$ of suspected patients had findings suggestive of COVID-19 in chest CT while the positive rate of RT-PCR kits was only $59 \%$ [9]. Of those with negative RT-PCR, CT findings were suggestive of highly likely disease and probable disease $48 \%$ and $33 \%$ respectively [9]. It was also shown that CT findings were detected in infected individuals before the RT-PCR kits did in symptomatic individuals. In patients whose initial serologic result was negative and subsequently were found to be positive on repeat testing, the second positive test results took an average of 5.1 days. Ninety-seven percent of those cases already had positive imaging findings, which were present with a mean of 8 days before the second positive serologic test results were available [9].

Bilateral ground-glass opacities (GGO) and/or consolidation have been suggested as the hallmark of COVID-19 infection on chest CT images [7, 9, 13-15]. While they may be nonspecific, these findings are easily identifiable on CT and should in the proper clinical setting aid diagnosis. GGO is an increased attenuation with preserved vascular and airway markings. With consolidation, these markings are obscured by the infiltration's effect on images. In a recent study, $78 \%$ of symptomatic patients had these findings on CT; of those, $60 \%$ demonstrated bilateral lung involvement. Imaging findings were detected in $91 \%$ of cases within 3-5 days after symptom onset. Interestingly, the serologic tests result became available at almost the same time in this study (4.5 days) [14].

\section{Low- and ultra-low-dose CT scan protocol}

In emergent situations such as COVID-19 pandemic, the demand for performing CT scans may significantly increase due to the high rate of infected individuals. Myers et al. have recently suggested that radiology departments should prepare for the flood of COVID-19 patients during the outbreak and therefore should be engaged in creating the hospital's mass casualty incident plan [16]. Furthermore, severely symptomatic patients will often undergo multiple imaging studies during the course of their illness. The cumulative effect of these multiple exams can significantly increase the cumulative radiation dose these patients may receive during their hospitalization and recovery. The as low as reasonably achievable (ALARA) principle states that whenever radiation is expected, the exposure should be as low as reasonably achievable [17]. Keeping this important principle in mind, it is critical that we remember that any CT scan we perform should be accompanied by the justification of examination and optimization of radiation dose [18]. This is of particular importance when evaluating more radiation-sensitive patient populations such as pregnant patients and children. While $\mathrm{CT}$ imaging has been shown to be a great help in establishing the diagnosis of COVID-19, the potential for increased radiation exposure to a large number of patients across the country cannot be ignored. Balancing the need for effective imaging to aid rapid diagnosis while also trying to minimize radiation exposure will be important for radiologists and clinicians alike as we work together to manage to COVID-19 outbreak.

Replacing conventional CT (CCT) with low-dose CT (LDCT) and ultra-low-dose CT (ULDCT) has been proposed as a method to decrease radiation exposure in these patients (Figs. 1 and 2). In a retrospective study [13], LDCT with iterative reconstruction (IR) demonstrated sensitivity, specificity, positive predictive value, negative predictive value, and accuracy of about $90 \%$ in the diagnosis of COVID-19. These parameters further increased to up to $96 \%$ when patients were symptomatic for more than $48 \mathrm{~h}$. The likelihood of COVID-19 diagnosis increased to $91 \%$ from pre-LDCT value of $43 \%$ in patients with suggestive imaging findings whereas it was decreased to $9 \%$ in those with normal or atypical imaging findings. The respective post-LDCT values in patients with $>48 \mathrm{~h}$ of symptoms were $91 \%$ and 3\%. Furthermore, LDCT demonstrated an additive diagnostic benefit in patients with concomitant bacterial pneumonia or an alternative diagnosis other than COVID-19.

Since GGO and consolidation are the primary CT presentations of COVID-19 that have been shown to be effectively detected in LDCT or ULDCT, these low-dose image acquisition methods can be utilized for the evaluation of more radiation-sensitive individuals such as pregnant patients. The radiation dose with chest CCT is estimated at $7 \mathrm{mSv}$, which is reduced to $1-1.5 \mathrm{mSv}$ with the new LDCT methods and as low as $0.3 \mathrm{mSv}$ with ULDCT ones [18]. LDCT and ULDCT have shown efficacy to detect lung abnormalities (Table 1). Park et al. suggested that utilization of LDCT in the evaluation of patients suspected to have lower respiratory infections had successfully detected GGO and small consolidation when these pathologic findings were not detectable in the radiographic images [22]. Kubo et al. compared the detection of GGO and reticular opacities of LDCT with a previous CCT 


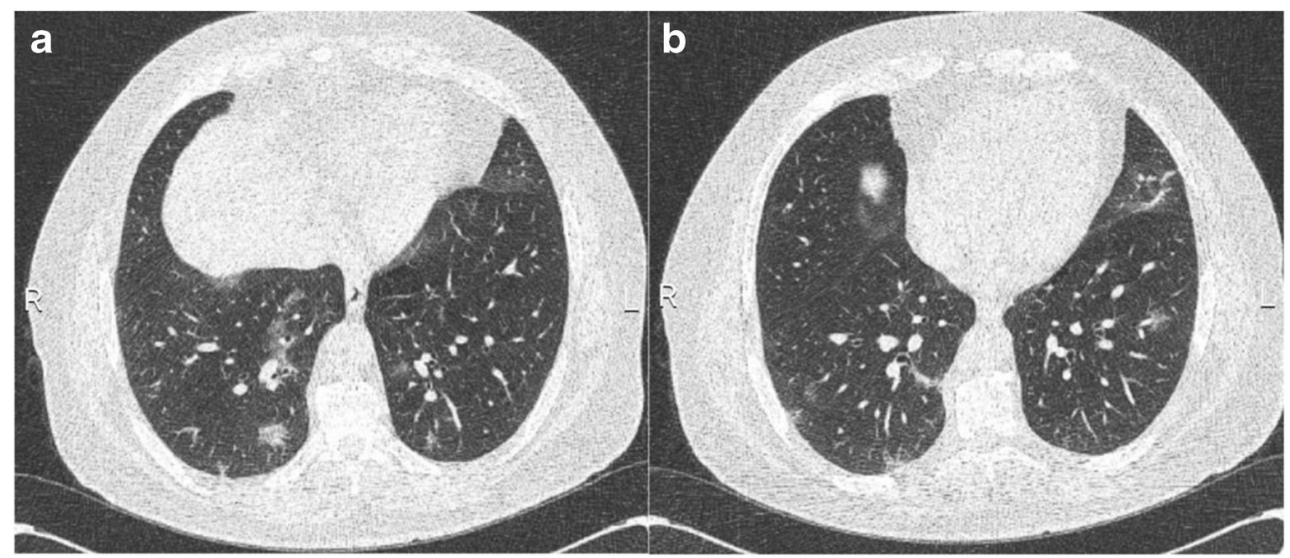

Fig. 1 A 57-year-old male presented with fever and shortness of breath in an epidemic area of COVID-19 (Iran) for diagnostic work-up. Low-dose CT demonstrates multifocal basilar predominant peripheral ground-glass opacities, characteristic of SARS-CoV-2 pneumonia $(\mathbf{a}, \mathbf{b})$. The low-dose $\mathrm{CT}$ chest radiation dosimetry of the patient was DLP of $78.34 \mathrm{mGy} \times \mathrm{cm}$ and CTDIvol of $2.03 \mathrm{mGy}$. Conventional CT chest protocol in the same

and reported an agreement rate of $83 \%$ between them [25]. In contrast, Christe et al. showed that sensitivity of LDCT (77\%) is less than CCT (89\%) in the detection of GGO particularly for patchy lesions but no significant difference was observed in the detection of consolidation between these scans. In fact, of the missed GGO lesion, half were detected as a nodule [26]. Kim Y. et al. observed no significant differences between LDCT and ULDCT in the detection of GGO and consolidation [19].

Likewise, pediatric patients benefit from dose reduction as they are at increased risk of malignancy following radiation due to their longer life expectancy and higher cell proliferation. A 1-year-old child is 10 times more susceptible to the adverse effects of ionizing radiation than an adult [28]. In a study published in JAMA, the routine effective dose delivered to pediatric patients during chest $\mathrm{CT}$ was calculated between 5 and $8 \mathrm{mAs}$. The lifetime attributable risk of solid organ malignancies per 10,000 CT scans was measured as high as 30 patient would result in DLP of $301.14 \mathrm{mGy} \times \mathrm{cm}$ and CTDIvol of $7.87 \mathrm{mGy}$, which means that low-dose protocol decreased the radiation dose to less than $50 \%$ without a significant impact on the diagnostic value. (CT protocol: $100 \mathrm{kVp}, 40 \mathrm{mAs}, 0.5 \mathrm{~s}$ without iterative reconstruction)

and 9 for girls and boys respectively. As it is expected, the projected radiation dose to body organs is higher in thyroid, lung, breast, and esophagus during a chest $\mathrm{CT}$ acquisition. It was reported that as low as $350 \mathrm{CT}$ scans are needed to be performed to observe 1 cancer in girls [29]. LDCT scan has been also suggested in the evaluation of lung abnormalities in pediatrics and successfully detected GGOs and consolidations. Sun et al. measured an overall radiation dose of $0.59 \mathrm{mSv}$ for evaluation of necrotizing pneumonia in different methods of irritative reconstruction which was significantly lower than CCT. They also demonstrated better detection and lower noise for lung lesions using LDCT performed with iterative reconstruction [21]. Therefore, low-dose CT image acquisition methods are of value in the evaluation of pediatric patients.

IR methods have been used to improve image quality in low-dose image acquisition and decrease noise while reducing radiation dose. Sakai et al. used simulated GGO and reticular
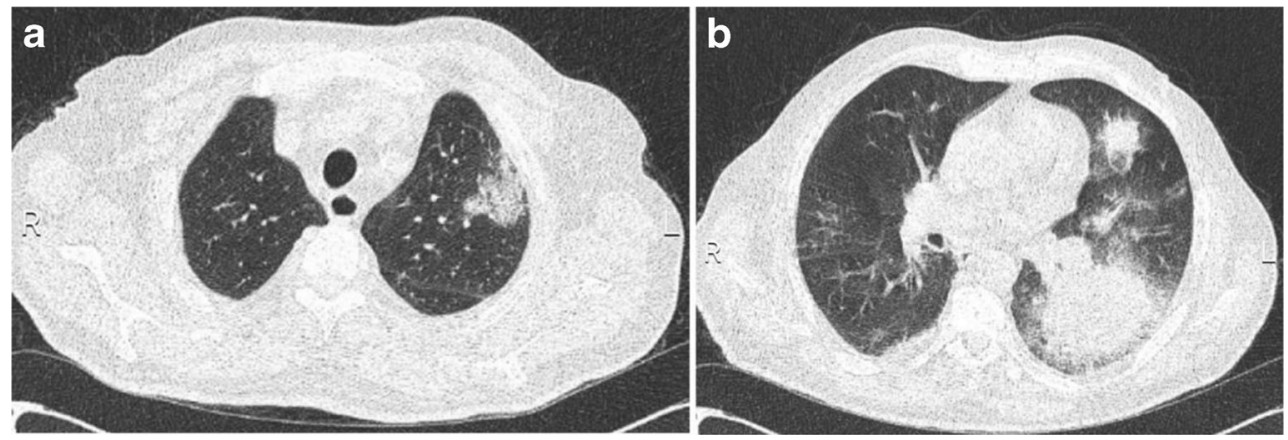

Fig. 2 Low-dose chest CT in a 77-year-old male with high-grade fever, shortness of breath, malaise, and cough in an epidemic area of COVID-19 (Iran) demonstrate left upper lobe ground-glass opacity (a). The patient had areas of consolidation superimposed on ground-glass opacity in bilateral lung bases (b). The constellation of findings is in keeping with SARS-CoV-2 pneumonia. The low-dose CT chest radiation dosimetry of the patient was DLP of $68.21 \mathrm{mGy} \times \mathrm{cm}$ and CTDIvol of $2.10 \mathrm{mGy}$. Conventional CT chest protocol in the same patient would result in DLP of $264.55 \mathrm{mGy} \times \mathrm{cm}$ and CTDIvol of $5.92 \mathrm{mGy}$, which means that low-dose protocol decreased the radiation dose to about $50 \%$ without a significant impact on the diagnostic value. (CT protocol: $100 \mathrm{kVp}$, $50 \mathrm{mAs}, 0.5 \mathrm{~s}$ without iterative reconstruction) 
Table 1 Studies assessed application of chest low-dose CT or ultra-low-dose CT in evaluation of pneumonia

\begin{tabular}{|c|c|c|c|c|}
\hline Study & Protocol & Effective dose & IR & Imaging output \\
\hline \multirow[t]{3}{*}{ Kim et al. (2014) [19] } & $\begin{array}{l}\text { LDCT } \\
120 \mathrm{kVp} ; 30 \mathrm{mAs}\end{array}$ & $1.06 \pm 0.11 \mathrm{mSv}$ & + & $\begin{array}{l}\text { There was no difference in detection of } \\
\text { consolidation and GGO. Diagnosis was clear in } \\
100 \% \text { of LDCT and } 96 \% \text { of ULDCT. }\end{array}$ \\
\hline & ULDCT A $100 \mathrm{kVp} ; 20 \mathrm{mAs}$ & $0.44 \pm 0.05 \mathrm{mSv}$ & + & \\
\hline & ULDCT B $80 \mathrm{kVp} ; 30 \mathrm{mAs}$ & $0.31 \pm 0.0 .3 \mathrm{mSv}$ & + & \\
\hline Dorneles et al. (2018) [20] & $\begin{array}{l}\text { ULDCT } \\
80 \mathrm{kVp} ; 15-30 \mathrm{mAs} ; 0.5 \mathrm{~s}\end{array}$ & $0.39 \pm 0.15 \mathrm{mSv}$ & + & $\begin{array}{l}\text { Image qualities were excellent or diagnostic in } 99 \% \\
\text { of ULDCTs. }\end{array}$ \\
\hline Sun et al. (2017) [21] & $\begin{array}{l}\text { LDCT } \\
120 \mathrm{kVp} ; 10-350 \mathrm{mAs} ; 0.8 \mathrm{~s}\end{array}$ & $0.59 \pm 0.19 \mathrm{mSv}$ & + & $\begin{array}{l}\text { IR demonstrated better detection of pulmonary } \\
\text { lesions and lesser noise than FBP. }\end{array}$ \\
\hline Park et al. (2015) [22] & $\begin{array}{l}\text { LDCT } \\
100 \mathrm{kVp} ; 30 \mathrm{mAs} ; 0.5 \mathrm{~s}\end{array}$ & Not reported & - & $\begin{array}{l}\text { LDCT detected GGO and small consolidation } \\
\text { which were undetected in radiograph. }\end{array}$ \\
\hline Dorobisz et al. (2017) [23] & $\begin{array}{l}\text { LDCT } \\
120 \mathrm{kVp} ; 25,50,75,100 \mathrm{mAs}\end{array}$ & $0.748-2.55 \mathrm{mSv}$ & + & $\begin{array}{l}\text { LDCT successfully detected GGO and } \\
\text { consolidation }\end{array}$ \\
\hline Alamdaran et al. (2019) [24] & $\begin{array}{l}\text { LDCT } \\
120 \mathrm{kVp} ; 30,50 \mathrm{mAs}\end{array}$ & Not reported & - & $\begin{array}{l}\text { Diagnoses of LDCTs were concordant with final } \\
\text { diagnoses. }\end{array}$ \\
\hline Kubo et al. (2016) [25] & $\begin{array}{l}\text { CCT } \\
120 \mathrm{kVp} ; 150 \mathrm{mAs} ; 0.5 \mathrm{~s} \\
\text { LDCT } \\
120 \mathrm{kVp} ; 150 \mathrm{mAs} ; 0.5 \mathrm{~s}\end{array}$ & $\begin{array}{l}10.7 \\
3.57\end{array}$ & - & $\begin{array}{l}\text { There were }>83 \% \text { concordance between CCT } \\
\text { findings and LDCT and no significant difference } \\
\text { in detection rate. }\end{array}$ \\
\hline Christe et al. (2012) [26] & $\begin{array}{l}\text { CCT } \\
120 \mathrm{mKv} ; 150 \mathrm{mAs} \\
\mathrm{LDCT} \\
120 \mathrm{mKv} ; 40 \mathrm{mAs}\end{array}$ & $\begin{array}{l}\text { Not reported } \\
\text { Not reported }\end{array}$ & - & $\begin{array}{l}\text { LDCT had lower sensitivity for GGO with no } \\
\text { significant difference for consolidation. }\end{array}$ \\
\hline Ohno et al. (2012) [27] & $\begin{array}{l}\text { CCT } \\
120 \mathrm{kVp} ; 150 \mathrm{mAs} ; 0.5 \mathrm{~s} \\
\text { LDCT A } \\
120 \mathrm{kVp} ; 50 \mathrm{mAs} ; 0.5 \mathrm{~s} \\
\text { LDCT B } \\
120 \mathrm{kVp} ; 25 \mathrm{mAs} ; 0.5 \mathrm{~s}\end{array}$ & $\begin{array}{l}\text { Not reported } \\
\text { Not reported } \\
\text { Not reported }\end{array}$ & $\begin{array}{l}- \\
\pm \\
\pm\end{array}$ & $\begin{array}{l}\text { Image quality of LDCT methods was significantly } \\
\text { low when IR was not applied. There was }>80 \% \\
\text { concordance between detection of GGO and } \\
\text { reticular opacities in all three methods. }\end{array}$ \\
\hline
\end{tabular}

$G G O$, ground-glass opacity; $C C T$, conventional CT; $L D C T$, low-dose CT; $U L D C T$, ultra-low-dose $\mathrm{CT}$; $I R$, iterative reconstruction; $F B P$, filtered back projection

opacity lesions and implemented them inside the apex and lower lobe of the lung [30]. They obtained LDCT images using the IR method in various levels of radiation dose and compared it with filtered back projection (FBP) method which is widely used in scanners. They observed that although there was no significant difference in visual detection of reticular opacity between these two modalities, IR dramatically improved GGO visualization at low radiation doses. In a clinical study, IR demonstrated better detection of necrotizing lesions in children evaluated for necrotizing pneumonia [21]. Ohno et al. showed that without the implementation of IR, the image quality of LDCT was significantly lower than CCT. Following the addition of IR, no significant difference was noted [27].

\section{Conclusion}

Since CT scan is capable to aid the diagnosis of COVID-19 in conjunction with other clinical findings, the utilization of LDCT and ULDCT with iterative reconstruction can be potentially recommended for the evaluation of these patients particularly in pregnant and pediatric populations to reduce radiation exposure. Although LDCT and ULDCT have demonstrated comparable efficacy in the detection of GGO and consolidation in patients with pneumonia, no studies have evaluated the efficacy of LDCT and ULDCT in the detection of pulmonary findings in the setting of COVID-19. A head to head comparison of low-dose and conventional protocol in very early stages of the disease is needed, while in intermediate and advanced stages, probably the low-dose CT protocol will provide adequate image quality and diagnostic accuracy.

Author contribution Authors contributed equally in all aspects of study.

Data availability The authors declare that they had full access to all of the data in this study and the authors take complete responsibility for the integrity of the data and the accuracy of the data analysis.

\section{Compliance with ethical standards}

Conflict of interest The authors declare that they have no conflict of interest. 


\section{References}

1. Coronavirus disease (COVID-2019) situation reports: World Health Organization (WHO); 2020 [updated 24 April 2020. Situation report-95:[Available from: https://www.who.int/ emergencies/diseases/novel-coronavirus-2019/situation-reports

2. Zhou P, Yang X-L, Wang X-G, Hu B, Zhang L, Zhang W et al (2020) A pneumonia outbreak associated with a new coronavirus of probable bat origin. Nature. https://doi.org/10.1038/s41586-0202012-7

3. Report of the WHO-China Joint Mission on Coronavirus Disease 2019 (COVID-19): World Health Organization (WHO); 2020 [Available from: https://www.who.int/docs/default-source/ coronaviruse/who-china-joint-mission-on-covid-19-final-report. pdf

4. COVID-19 Map - Johns Hopkins Coronavirus Resource Center: Johns Hopkins University (JHU); 2020 [updated 24 April 2020. Available from: https://coronavirus.jhu.edu/map.html

5. Holshue ML, DeBolt C, Lindquist S, Lofy KH, Wiesman J, Bruce $\mathrm{H}$ et al (2020) First case of 2019 novel coronavirus in the United States. N Engl J Med. https://doi.org/10.1056/NEJMoa2001191

6. Huang C, Wang Y, Li X, Ren L, Zhao J, Hu Y, Zhang L, Fan G, Xu J, Gu X, Cheng Z, Yu T, Xia J, Wei Y, Wu W, Xie X, Yin W, Li H, Liu M, Xiao Y, Gao H, Guo L, Xie J, Wang G, Jiang R, Gao Z, Jin Q, Wang J, Cao B (2020) Clinical features of patients infected with 2019 novel coronavirus in Wuhan, China. Lancet 395(10223):497506

7. Hosseiny M, Kooraki S, Gholamrezanezhad A, Reddy S, Myers L (2020) Radiology perspective of coronavirus disease 2019 (COVID-19): lessons from severe acute respiratory syndrome and Middle East respiratory syndrome. AJR Am J Roentgenol 1-5

8. Kooraki S, Hosseiny M, Myers L, Gholamrezanezhad A (2020) Coronavirus (COVID-19) outbreak: what the Department of Radiology should know. J Am Coll Radiol. S1546440(20)30150-2

9. Ai T, Yang Z, Hou H, Zhan C, Chen C, Lv W, et al. (2020) Correlation of chest $\mathrm{CT}$ and RT-PCR testing in coronavirus disease 2019 (COVID-19) in China: a report of 1014 cases. Radiology. 200642

10. Willman D Contamination at CDC lab delayed rollout of coronavirus tests - The Washington Post 2020 [Available from: https://www. washingtonpost.com/investigations/contamination-at-cdc-labdelayed-rollout-of-coronavirus-tests/2020/04/18/fd7d3824-713911ea-aa80-c2470c6b2034_story.html

11. Restuccia A Manufacturing defect in some early CDC test kits being probed: The Wall Street Journal (WSJ); 2020 [Available from: https://www.wsj.com/articles/manufacturing-defect-in-someearly-cdc-test-kits-being-probed-11583119414

12. Gates B (2020) Responding to Covid-19 - a once-in-a-century pandemic? N Engl J Med. https://doi.org/10.1056/NEJMp2003762

13. Dangis A, Gieraerts C, Bruecker YD, Janssen L, Valgaeren H, Obbels D et al (2020) Accuracy and reproducibility of low-dose submillisievert chest CT for the diagnosis of COVID-19. Radiol Cardiothorac Imaging 2(2):e200196

14. Bernheim A, Mei X, Huang M, Yang Y, Fayad ZA, Zhang N, et al. (2020) Chest CT findings in coronavirus disease-19 (COVID-19): relationship to duration of infection. Radiology. 200463

15. Salehi S, Abedi A, Balakrishnan S, Gholamrezanezhad A (2020) Coronavirus disease 2019 (COVID-19): a systematic review of imaging findings in 919 patients. AJR Am J Roentgenol 1-7
16. Myers L, Balakrishnan S, Reddy S, Gholamrezanezhad A (2020) Coronavirus outbreak: is radiology ready? Mass casualty incident planning. J Am Coll Radiol

17. Bell DJ, Gerstmair A As low as reasonably achievable (ALARA): radiopaedia.org; [Available from: https://radiopaedia.org/articles/ as-low-as-reasonably-achievable-alara?lang=us

18. Beregi JP, Greffier J (2019) Low and ultra-low dose radiation in CT: opportunities and limitations. Diagn Interv Imaging 100(2):63-64

19. Kim Y, Kim YK, Lee BE, Lee SJ, Ryu YJ, Lee JH, Chang JH (2015) Ultra-low-dose CT of the thorax using iterative reconstruction: evaluation of image quality and radiation dose reduction. AJR Am J Roentgenol 204(6):1197-1202

20. Dorneles CM, Pacini GS, Zanon M, Altmayer S, Watte G, Barros MC, Marchiori E, Baldisserotto M, Hochhegger B (2020) Ultralow-dose chest $\mathrm{CT}$ without anesthesia in the assessment of pediatric pulmonary diseases. J Pediatr 96(1):92-99

21. Sun J, Yu T, Liu J, Duan X, Hu D, Liu Y et al (2017) Image quality improvement using model-based iterative reconstruction in low dose chest $\mathrm{CT}$ for children with necrotizing pneumonia. BMC Med Imaging 17(1):24

22. Park JE, Kim Y, Lee SW, Shim SS, Lee JK, Lee JH (2016) The usefulness of low-dose CT scan in elderly patients with suspected acute lower respiratory infection in the emergency room. Br J Radiol 89(1060):20150654

23. Zaleska-Dorobisz U, Olchowy C, Łasecki M, Sokołowska-Dąbek D, Pawluś A, Frączkiewicz J, Gorczyńska E (2017) Low-dose computed tomography in assessment of pulmonary abnormalities in children with febrile neutropenia suffering from malignant diseases. Adv Clin Exp Med 26(4):695-701

24. Alamdaran A, Dana M, Hashemi J, Morovatdar N, Sayedi SJ (2020) Clinical value of low dose CT- scan in pediatric chest diseases: adequacy assessment. Int J Pediatr 8(2):10845-10851

25. Kubo T, Ohno Y, Nishino M, Lin P-J, Gautam S, Kauczor H-U, Hatabu H, iLEAD study group (2016) Low dose chest CT protocol $(50 \mathrm{mAs})$ as a routine protocol for comprehensive assessment of intrathoracic abnormality. Eur J Radiol 3:86-94

26. Christe A, Charimo-Torrente J, Roychoudhury K, Vock P, Roos JE (2013) Accuracy of low-dose computed tomography (CT) for detecting and characterizing the most common CT-patterns of pulmonary disease. Eur J Radiol 82(3):e142-ee50

27. Ohno Y, Takenaka D, Kanda T, Yoshikawa T, Matsumoto S, Sugihara N, Sugimura K (2012) Adaptive iterative dose reduction using $3 \mathrm{D}$ processing for reduced- and low-dose pulmonary CT: comparison with standard-dose CT for image noise reduction and radiological findings. AJR Am J Roentgenol 199(4):W477-WW85

28. Pearce MS, Salotti JA, Little MP, McHugh K, Lee C, Kim KP, Howe NL, Ronckers CM, Rajaraman P, Craft AW, Parker L, Berrington de González A (2012) Radiation exposure from CT scans in childhood and subsequent risk of leukaemia and brain tumours: a retrospective cohort study. Lancet. 380(9840):499-505

29. Miglioretti DL, Johnson E, Williams A, Greenlee RT, Weinmann S, Solberg LI, Feigelson HS, Roblin D, Flynn MJ, Vanneman N, Smith-Bindman R (2013) The use of computed tomography in pediatrics and the associated radiation exposure and estimated cancer risk. JAMA Pediatr 167(8):700-707

30. Sakai N, Yabuuchi H, Kondo M, Matsuo Y, Kamitani T, Nagao M et al (2015) Low-dose CT screening using hybrid iterative reconstruction: confidence ratings of diagnoses of simulated lesions other than lung cancer. Br J Radiol 88(1053):20150159

Publisher's note Springer Nature remains neutral with regard to jurisdictional claims in published maps and institutional affiliations. 